\title{
Complex chemistry in star-forming regions
}

\author{
Robin T. Garrod ${ }^{1}$, Susanna L. Widicus Weaver ${ }^{2}$, and Eric Herbst ${ }^{3}$ \\ ${ }^{1}$ Max-Planck-Institut für Radioastronomie \\ Auf dem Hügel 69, 53121 Bonn, Germany \\ email: rgarrod@mpifr-bonn.mpg.de \\ ${ }^{2}$ Depts. of Chemistry \& Astronomy, University of Illinois at Urbana-Champaign \\ Urbana, IL 61801, USA \\ email: slww@uiuc.edu \\ ${ }^{3}$ Departments of Physics, Chemistry and Astronomy, The Ohio State University \\ Columbus, OH 43210, USA \\ email: herbst@mps . ohio-state.edu
}

\begin{abstract}
We present a new gas-grain chemical model that allows the grain-surface formation of saturated, complex, organic species from their constituent functional-groups-basic building blocks that derive from the cosmic ray-induced photodissociation of the granular ice mantles. The surface mobility of the funtional-group radicals is crucial to the reactions, and much of the formation of complex molecules occurs at the intermediate temperatures $(\sim 20-40 \mathrm{~K})$ attained during the warm-up of the hot core. Our model traces the evolution of a large range of detected, and as yet un-detected, complex molecules.
\end{abstract}

Keywords. Astrochemistry, ISM: molecules, stars: formation

Hot cores are dense $\left(n_{\mathrm{H}} \simeq 10^{7} \mathrm{~cm}^{-3}\right)$, hot $\left(T_{\mathrm{K}} \geqslant 100 \mathrm{~K}\right)$ regions associated with high-mass star formation. Their $\mathrm{mm} / \mathrm{sub}-\mathrm{mm}$ spectra are rich, and typically indicate large abundances $\left(\sim 10^{-8} n_{\mathrm{H}}\right)$ of complex organic molecules like formic acid (HCOOH), methyl formate $\left(\mathrm{HCOOCH}_{3}\right)$, dimethyl ether $\left(\mathrm{CH}_{3} \mathrm{OCH}_{3}\right)$, and ethanol $\left(\mathrm{CH}_{3} \mathrm{CH}_{2} \mathrm{OH}\right)$ (van Dishoeck \& Blake 1998). In the past, gas-phase chemistry was thought to be capable of producing complex organics, but new experimental (Horn et al. 2004) and theoretical studies (Geppert et al. 2006) suggest this is typically not the case, pointing towards a much stronger role for dust-grain surface chemistry in star-forming regions.

Here we present a fully-coupled gas-grain chemical model (Garrod et al. 2008), in which simple radicals may combine on grain surfaces to form much larger complex molecules. We use a greatly extended grain-surface chemical network, based on the model of Garrod \& Herbst (2006). We allow surface reactions between all of the species $\mathrm{H}, \mathrm{OH}, \mathrm{CO}, \mathrm{HCO}$, $\mathrm{CH}_{3}, \mathrm{CH}_{3} \mathrm{O}, \mathrm{CH}_{2} \mathrm{OH}, \mathrm{NH}$, and $\mathrm{NH}_{2}$. The radicals are mainly produced by cosmic rayinduced photodissociation of the main constituents of the icy dust-grain mantles. As the hot-core temperature increases, the heavier radicals become mobile, and therefore reactive. Figure 1 shows four organic molecules that have been detected in hot cores, and the four simple functional-group radicals from which they may be formed on the grain surfaces. As temperatures approach $100 \mathrm{~K}$, grain-surface species begin to evaporate. Gas-phase ion-molecule destruction mechanisms are included in the network for all new species. In all, 50 neutrals and 32 ionic species have been added.

We have run gas-grain chemical models of the cold collapse phase and subsequent warm-up phase $(10 \rightarrow 200 \mathrm{~K})$ of a hot core. The chemistry of the gas phase and grain surfaces are fully-coupled, using a rate-equation treatment. The post-collapse gas density is $10^{7} \mathrm{~cm}^{-3}$. Figure 2 shows abundances of some complex organic molecules, during an intermediate-length hot-core warm-up phase. Large amounts of methyl formate, 


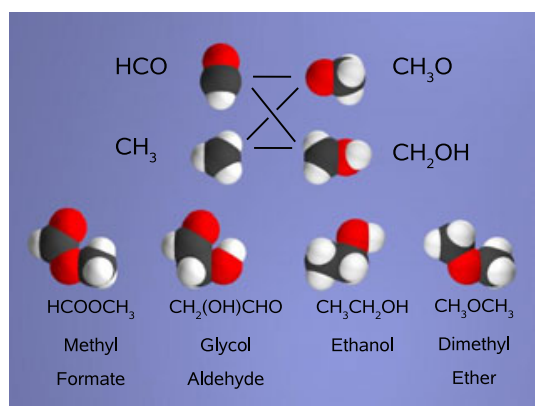

Figure 1. Four complex molecules, and the functional groups from which they may form on dust-grain surfaces during the hot-core warm-up phase.
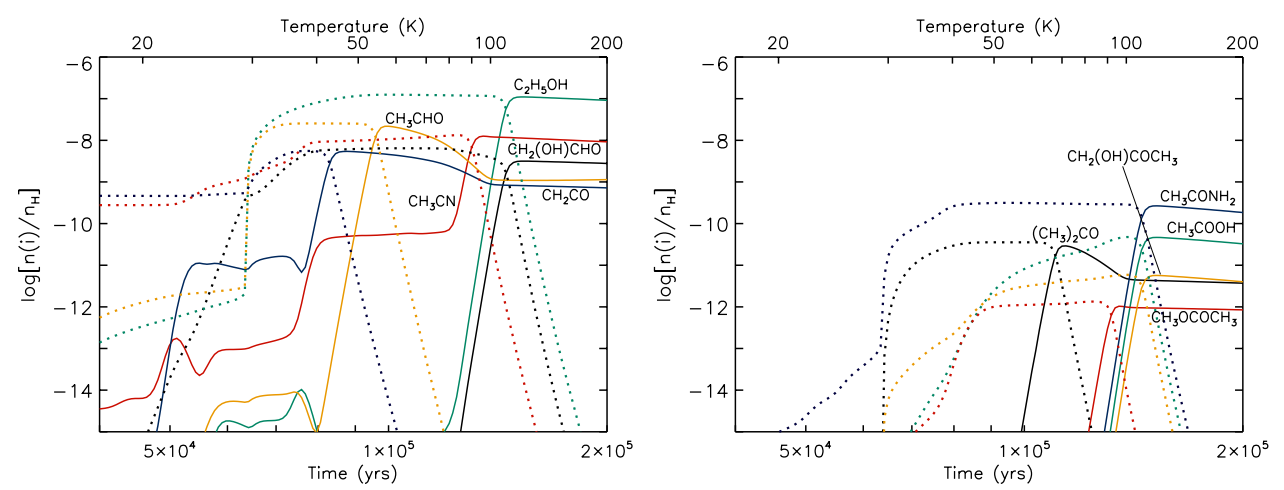

Figure 2. Modelled molecular abundances during the hot-core warm-up phase. Solid lines indicate gas-phase species; dotted lines of the same colour indicate the grain-surface species.

dimethyl ether, glycolaldehyde and ethanol, as well as other molecules like acetaldehyde $\left(\mathrm{CH}_{3} \mathrm{CHO}\right)$, are formed at $\sim 30-40 \mathrm{~K}$, when the radicals $\mathrm{CH}_{3}$ and $\mathrm{HCO}$ become mobile on the grains. Dimethyl ether is still formed most strongly in the gas phase.

In summary, we find that complex organic molecules may be formed efficiently on grain-surfaces by the addition of heavy, functional-group radicals derived from the icy mantles. The various abundances of structural isomers may be explained by different formation routes. The evaporation of certain species at intermediate temperatures $(\sim 40-$ $50 \mathrm{~K}$ ) agrees well with observed rotational temperatures of such molecules, implying a strong interaction between grain-surface and gas-phase processes. While some species are formed on the grains, other species (including dimethyl ether, $\mathrm{CH}_{3} \mathrm{OCH}_{3}$ ) are still formed in the gas phase as a result of the evaporation of ices. This may occur at any temperature at which important species evaporate-not just at the canonical hot-core temperature of $100 \mathrm{~K}$ or so. Species of greater complexity, e. g. acetone, acetamide, are formed as a result of the break-down and re-construction of smaller complex molecules. The longer timescales required for these processes make high-mass star-forming regions more favourable to greater molecular complexity.

\section{References}

Garrod, R. T. \& Herbst, E. 2006, AA, 457, 927

Garrod, R. T., Widicus Weaver, S. L., \& Herbst, E. 2008, ApJ, 682, 283

Geppert, W. D., Hamberg, M., Thomas, R. D., et al. 2006, Faraday Discuss., 133, 51

Horn, A., Møllendal, H., Sekiguchi, O., et al. 2004, ApJ, 611, 605

van Dishoeck, E. F. \& Blake, G. A. 1998, ARAA, 36, 317 